\title{
Power re-allocation for reducing contracted electric power costs
}

\author{
F. Torrent-Fontbona ${ }^{\mathrm{a}, *}$, B. López ${ }^{\mathrm{a}}$ \\ ${ }^{a}$ eXiT research group, Institute of Informatics and Applications, University of Girona, \\ Campus Montilivi, Building P4, 17071 Girona
}

\begin{abstract}
Electric bills consist of a cost related to the consumed energy and a cost related to the maximum demanded power. This latter part usually accounts for approximately $25 \%$ to $40 \%$ of the bill. Demanded power by big consumers is measured in real time and electric companies highly penalise them if the maximum demanded power (along the billing period) exceeds the contracted power by the consumer. In this paper we propose a new method that, given the demanded power of close consumers for a time window (power profile), power costs are reduced by re-allocating the demanded power among consumers in order to keep all of them below or equal to their contracted power. We also propose and analyse some strategies to set a preference when not all power profiles can be kept below the contracted power. We tested this method using real-based simulated power profiles of eight different business buildings located in Girona and the power cost reduction achieved reached approximately $20 \%$.
\end{abstract}

Keywords: Electric power, allocation, smart grid, micro grid, virtual power plant, electricity, fairness

\footnotetext{
${ }^{*}$ Corresponding author

Email addresses: ferran.torrent@udg.edu (F. Torrent-Fontbona), beatriz.lopez@udg.edu (B. López)

URL: http://eia.udg.es/ ftorrent (F. Torrent-Fontbona), http://eia.udg.es/〜blopez (B. López)
} 


\section{Introduction}

Electric companies charge their customers for the amount of energy demanded and for the contracted power they have. Contracted power is supposed to be the maximum power at which the electric service will be interrupted (by some physical device) if the power required by the customer exceeds it. However, the popularisation of maximeters (devices that measure the maximum demanded power) has brought about electric tariffs that do not interrupt the electric service. Instead, these meters allow electric companies to apply maximum-power-dependent prices. For example, electric companies apply different prices depending on whether the demanded power of the customer exceeds the contracted power, or whether it is lower than a particular percentage of this contracted power.

Power cost is related to the infrastructure costs of electricity distribution companies. Trading companies use (and pay for) the infrastructure and in turn charge their customers for providing the required service (providing energy). As a consequence, trading companies have no margin to decrease the power cost (they are set by distribution companies), but they are interested in advising their customers on how they could reduce this part of their electricity bill.

Usually, maximum-power-dependent pricing is a demand-response strategy used by electricity companies which highly penalises the customer when it exceeds its contracted power even if it does so for a short period of time. For this reason we propose a new method called PRA (Power Re-Allocation) whereby customers consent to be assigned demanded power from others in order to keep all of them below the contracted power. So customers that do not use all of their contracted power transfer their surplus to neighbours who do exceed it. Therefore, power costs are reduced without reducing the sum of demanded power by all customers; it just re-allocates the demand among them. We also present some strategies to establish which customers have priority when not all can be put below the contracted power.

The benefits of using the PRA method compensates from large the investment required to implement the approach, mainly, individual customers converted to a single one, in a close distance.

This paper is organised as follows: first we present some work related to cost reduction to deal with demand response strategies; second we formulate the problem of minimising power costs under maximum-power-dependent prices; then we explain our method called PRA; next we present our experi- 
ments and the results obtained; finally we expose the conclusions of the work and propose some further work.

\section{Related Work}

A great deal of current literature concerns applications to reduce energy costs from the System Operator's (SO) point of view. For example Mohsenian-Rad et al. [13] present a new approach of demand-side management (including several consumers) based on game-theoretic energy consumption scheduling under variable prices (time-dependent and volume-dependent). It is a demand-side management approach that assumes that consumers will give their best response to price changes re-scheduling their consumption profile. Also from the SO point of view, Jia et al. [6] establish a multi-agent system to represent the interactivity between $\mathrm{SO}$ and terminal consumers. The authors propose a continuous analysis on demand response using strategies that they present, which reflect the interactive response between the SO and terminal consumers. Faria et al. [3] present an approach based on particle swarm optimisation to manage demand response on simulated power systems. Moreover, Ketter et al. [7] describe an energy market simulator used to study the dynamics of customer and retailer decisionmaking. Our work does not concern the SO but a coalition of customers. In this sense this paper proposes a methodology to reduce power costs from the terminal consumer point of view.

The concept of creating coalitions of energy consumers has been also proposed by Vinyals et al. [17]. They propose that consumers can create a coalition of consumers through their contacts in a social media network. The aim of the coalitions is to flatten their aggregated energy consumption profile and then allow consumers to buy energy together. Our work differs from Vinyals et al. [17] because we focus on costs related to the maximum demanded power by the consumer and in doing so consumers of the coalitions must be close geographically due to power constraints. Furthermore, we propose a centralised approach to manage coalitions while Vinyals et al. [17] propose a decentralised approach without a technological solution. In addition to energy or power issues, Leng et al. [10] analyse space-exchange problems between retailers. This work shares with our paper the concept of exchanging a good that is sometimes in excess, without involving monetary units. However, the application field is completely different and thus, the features of the problem. 
From consumers' point of view, Torrent-Fontbona \& López [16] present and analyse some approaches of resource allocation for minimising costs under time-dependent and volume-dependent energy prices in a project scheduling problem (assigning energy consuming resources to activities). Simonis \& Hadzic [15] calculate some lower limits for solving the workflow scheduling problem under time-dependent prices. Our work is complementary to these demand response applications because we propose that some consumers in the same zone, that have already scheduled their activities in order to minimise energy costs, share their power rights to keep all of them below their contracted power. Also, from the consumer point of view, there are many works like Zhang et al. [18] that present methodologies to optimise energy costs when the consumer has a particular energy generation capacity.

Focusing on the private sector, there are several companies such as Arista Power (US), MeasurLogic (US), EnerNoc (US) or Circuitor (Spain), that offer technological solutions for reducing power costs. These solutions are based on using storage systems and controlling and managing loads (disconnecting non-critical loads when the consumer surpasses the contracted power). However, there is not any solution based on making coalitions of consumers and taking advantage of the different power profiles to reduce power costs as we propose in this paper.

There are some works that analyse household behaviour regarding energy consumption and energy prices as Gottwalt et al. [5]; Brounen et al. [2] and introduce new indexes to measure demand response and models of consumers [8]. For further literature of demand-side management, Law et al. [9] describe the key objectives of demand-side management and surveys demand response architectures.

\section{Problem modelling}

In recent years the problem of determining the power cost of a customer has been changed due to the smart grid. The use of maximeters allows electricity companies to charge consumers for their maximum demanded power $m$, along a time window $W$. However, electricity companies penalise customers when $m$ exceeds the contracted power $c$. For example, in Spain, when $m<0.85 c$ the electric company charges for $85 \%$ of $c$; when $0.85 c \leq m \leq 1.05 c$ the company charges for $m$; and when $m>1.05 c$ the company charges for $m+2(m-1.05 c)$. Moreover, electric companies apply different billing periods that consist of classifying the demanded power according to the time-slot 
Table 1: Notation

\begin{tabular}{ll}
\hline$t$ & time index \\
$k$ & period index \\
$i$ & customer index \\
$W$ & time length between two bills \\
$N_{p}$ & number of periods \\
$N_{c}$ & number of customers \\
$c_{i, k}$ & contracted power by customer $i$ for period $k$ \\
$c_{k}^{u}$ & aggregated contracted power of the umbrella entity for period $k$ \\
$\alpha_{i, k}$ & under-power demand parameter \\
$\beta_{i, k}$ & over-power demand parameter \\
$p_{i, k, t}$ & demanded power by customer $i$ in period $k$ at time $t$ \\
$p_{i}$ & power profile \\
$m_{i, k}$ & maximum demanded power by customer $i$ along period $k$ \\
$m_{k}^{u}$ & aggregated maximum demanded power by the umbrella entity \\
$\pi_{i, k}$ & power price $(€ /$ kW) of customer $i$ for period $k$ \\
$\tau_{i, k}$ & target power of customer $i$ for period $k$ \\
$A P R_{k, t}$ & accumulated power rights by all customers in period $k$ and at time $t$ \\
$A D P_{k, t}$ & accumulated demanded power by all customers in period $k$ and at time $t$ \\
$P S_{k, t}$ & power sharing (capacity of all customers to accept power from others) at \\
& time $t$ of period $k$ \\
priority $_{i, k}$ & priority value of customer $i$ for period $k$ \\
$x_{i, k}$ & amount of time customer $i$ has received power from other in period $k$ \\
$z_{i, k, t}$ & amount of power customer $i$ has received from others at time $t$ of period $k$ \\
\hline
\end{tabular}


(time of the day) it is required. In this way, each period represents a particular part of every day, i.e. from 00:00 to 08:00. Continuing with the Spanish example, Spanish law says that each day must be divided in three periods, and therefore, there must be a maximum demanded power for each one.

In the general case, the payment or power costs of a customer can be formalised according to the following notation.

Customer: $i$ is the customer index $\left(i \in\left[1, N_{c}\right]\right)$;

Time window: $W$, time duration between two bills of a set of customers (usually a month).

Period: $k$, fraction of a day corresponding to a power tariff; $k \in\left[1, N_{p}\right] ; N_{p}$ is the number of periods which divide each day;

Slot: $t$, is the time index in a period; $t \in\left[1, \frac{W}{N_{p}}\right]$

Contracted power: $c_{i, k}$, the contracted power that gives the customer $i$ the rights of demanding up to $\beta_{i, k} c_{i, k}(\mathrm{~kW})$ in period $k$ without paying extra charges. $\alpha_{i, k} c_{i, k}$ is the minimum power to pay for.

\section{Under-power demand parameter: $\alpha_{i, k}$.}

Over-power demand parameter: $\beta_{i, k}$.

Demanded power: $p_{i, k, t}$, the demanded power of customer $i$ in period $k$ at time $t$.

Power profile: $p_{i}=\left\{p_{i, k, t}, \forall k, t\right\}$, is the power of any customer $i$ in a given time window (see for example p1 in Figure 1).

Maximum demanded power: $m_{i, k}$, the maximum demanded power $(\mathrm{kW})$ of customer $i$ along all $k$ periods of the time window $W$. It is calculated as

$$
m_{i, k}=\max _{t}\left(p_{i, k, t}\right)
$$

It is important to point out that a unique high value of a slot $t$ determines the maximum power, compromising the power costs of the whole period. 
Power price: $\pi_{i, k}$, the power price $(€ / \mathrm{kW})$ for the period $k .^{1}$

In such a way, the power cost of consumer $i$ for period $k$ can be computed as follows:

$$
\operatorname{cost}\left(m_{i, k}\right)= \begin{cases}\alpha_{i, k} c_{i, k} \cdot \pi_{i, k} & m_{i, k}<\alpha_{i, k} c_{i, k} \\ m_{i, k} \cdot \pi_{i, k} & \alpha_{i, k} c_{i} \leq m_{i, k} \leq \beta_{i, k} c_{i, k} \\ \left(m_{i, k}+2\left(m_{i, k}-\beta_{i, k} \cdot c_{i, k}\right)\right) \cdot \pi_{i, k} & \beta_{i, k} c_{i, k}<m_{i, k}\end{cases}
$$

In this scenario, consumers with $m_{i, k}<\alpha_{i, k} \cdot c_{i, k}$ are paying more than the power they demand and those with $\beta_{i, k} \cdot c_{i, k}<m_{i, k}$ are highly penalised for exceeding their contracted power. Thus, in a given group of customers, those that do not exceed $\alpha_{i, k} \cdot c_{i, k}$ could be interested in sharing their power rights with those that do exceed $\alpha_{i, k} \cdot c_{i, k}$ (specially those that exceed $\beta_{i, k} \cdot c_{i, k}$ ). One day a customer could be on the giving side, when in another it could be on the receiving side, obtaining mutual benefits over the long term.

Despite our starting point is the Spanish electricity billing, it can be generalised to other electricity billing based on charging consumers according to their demand peaks. For example, the power re-allocation problem stated in this paper is extensible to other countries such as Germany, the United Kingdom, Austria, Czech Republic, etc. In general, we can say that most countries use a billing methodology based on the demand of the consumers, but they differ in the types of consumers (big consumers, special consumers, small consumers, etc.) they apply these tariffs, the weight of demand charges on the whole price of the electricity services, the use of time dependent prices, the division of the billing in periods, etc.

The power re-allocation problem consists of finding the $m_{i, k}$ that minimises the consumers costs, that is,

$$
\min _{i, k} \operatorname{cost}\left(m_{i, k}\right)
$$

\section{Methodology}

This paper proposes a method (PRA) to re-allocate demanded power among customers close to each other, so that they can reduce their power

\footnotetext{
${ }^{1}$ Power prices $(€ / \mathrm{kW})$ differ from energy consumption prices $(€ / \mathrm{kWh})$ which can also be time-dependent (see [16]) for how to deal with energy consumption).
} 
costs. In this way, the method consists of re-allocating demanded power from those customers that exceed $\alpha_{i, k} \cdot c_{i, k}$, and preferably those that exceed $\beta_{i, k} \cdot c_{i, k}$, to those customers that do not exceed the minimum power, $\alpha_{i, k} \cdot c_{i, k}$. Figure 1 illustrates power re-allocation between two customers.

In doing so, we propose an umbrella entity which aggregates the demanded power of all of its customers and agrees a single contract with the power company (see Figure 2). Our proposal is that electricity trade companies offer their customers to manage the umbrella entity without an extra cost for the consumers. Thus, we think they can offer this service to catch customers thanks to its economic benefits, and we think that they can offer it free because the cost of running this service is negligible compared to current costs of electricity services: collecting consumers power data is carried out by already (or being) implanted smart meters and an extra smart meter (300) for the umbrella entity. Furthermore, we think electricity companies can offer this service free as they are offering currently services to optimise the contracted power (adjusting it to the consumption).

Regarding the umbrella entity, it internally re-allocates the demanded power and computes the costs of such demands, which are finally paid to the distribution company. If the demand peaks do not occur simultaneously, the total cost would be less than the sum of costs separately. In this sense, consumers would be able to reduce their aggregated demanded power while flattening the load of the grid with respect to the contracted power. This benefits distribution companies because they must provide an infrastructure able to support these contracted powers and this would avoid having to increase the infrastructure by using it more efficiently. In this sense, PRA can be used as a tool for increasing network usage, which is one of the main drawbacks (poor network usage) of using an electricity billing method based on demand charge. Figure 2 illustrates the relationship between the different agents involved. Note that it is not the same as a microgrid or a virtual power plant $[1 ; 14]$.

PRA can also be seen as an incentive for locally smooth the electricity demand, one of the main objectives for the future smart grid. Smoothing of the demand conveys a reduction of reserve generators which are usually active, but disconnected, waiting for covering a peak of demand. Then, a reduction of the active generators, and even a reduction of the installed generators may help to decrease the global need of energy and the overexploitation of the Earth with its climatic consequences. Thus, PRA can be added to other activities aimed to smooth and reduce power demand such 


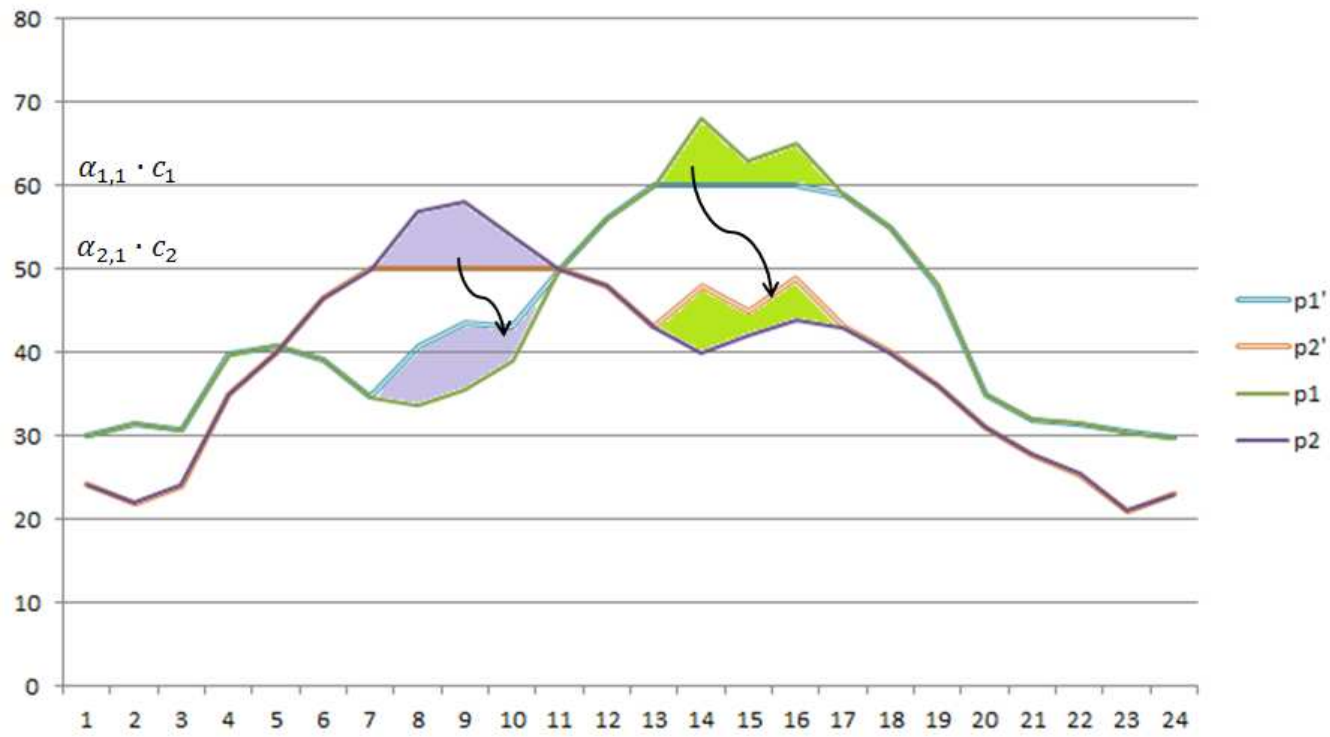

Figure 1: Illustration of how PRA re-allocate the excess of power of some consumers to others. Here, $p 1$ and $p 2$ represent the original power profiles and $p 1^{\prime}$ and $p 2^{\prime}$ represent the respective power profiles after power re-allocation.

as energy efficiency measures. See Meir \& Pearlmutter [11]; Meir et al. [12] for architectural energy efficiency measures and consequences of the climatic change.

Next, we describe how the aggregation is carried out in the umbrella entity, and we follow with the re-allocation algorithm descriptions and strategies.

\subsection{Umbrella entity}

The umbrella entity demands power equal to the sum of the power demands of the consumers behind it. Then this umbrella entity pays the distribution company according to this aggregated demanded power, taking into account, the maximum demanded power behaviour. On the one hand, it agrees a power contract for period $k, c_{k}^{u}$, equal to the sum of the contracted powers,

$$
c_{k}^{u}=\sum_{i} c_{i, k}
$$




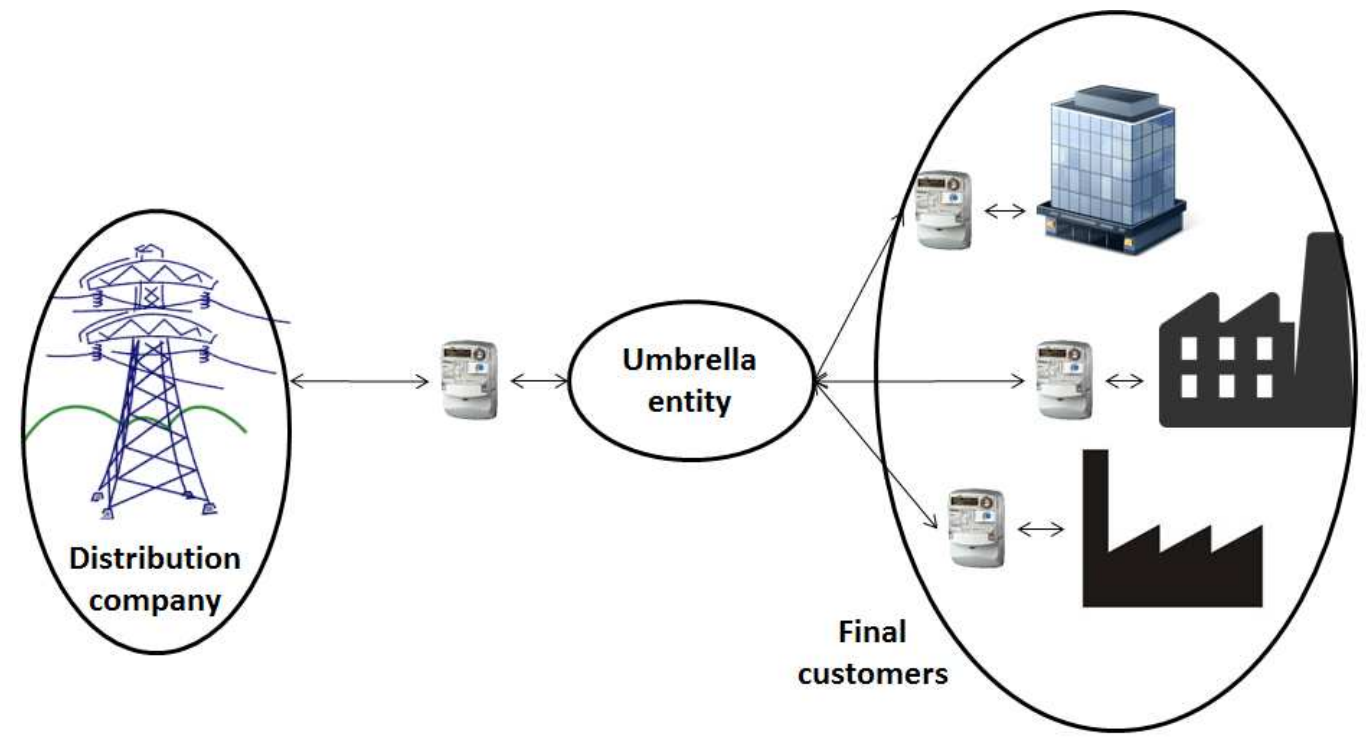

Figure 2: Illustration of the structure of the relationship between the different entities

On the other hand, the maximum demanded power by the umbrella entity, $m_{k}^{u}$ at a given period $k$ is

$$
m_{k}^{u}=\max _{t}\left\{\sum_{i} p_{i, k, t}\right\}
$$

Given the under and over power demand parameters for the umbrella entity, $\alpha_{k}$ and $\beta_{k}$, the payment for the umbrella entity is,

$$
\operatorname{cost}\left(m_{k}^{u}\right)= \begin{cases}\alpha_{k} c_{k}^{u} \cdot \pi_{k} & m_{k}^{u}<\alpha_{i, k} c_{k}^{u} \\ m_{k}^{u} \cdot \pi_{i, k} & \alpha_{k} c_{k}^{u} \leq m_{k}^{u} \leq \beta_{k} c_{k}^{u} \\ \left(m_{k}^{u}+2\left(m_{k}^{u}-\beta_{k} \cdot c_{k}^{u}\right)\right) \cdot \pi_{i, k} & \beta_{k} c_{k}^{u}<m_{k}^{u}\end{cases}
$$

For example, consider an umbrella entity that aggregates the consumption of two consumers like Figure 3. Both consumers have the same contracted profile which is registered in three periods $(k=1, \ldots, 3)$, of equal length, all of them are: $c_{i, 1}=40 \mathrm{~kW}$ (time slots 1 to 3 ), $c_{2,2}=50 \mathrm{~kW}$ (time slots 4 to 6 ), and $c_{i, 3}=30 \mathrm{~kW}$. The resulting aggregated contracted power for the umbrella entity is then, $c_{1}^{u}=80 k W, c_{2}^{u}=100 k W, c_{3}^{u}=60 k W$. For $t=2$, consumer one demands $45 \mathrm{~kW}$ going above its contracted power $\left(c_{1,1}=40 \mathrm{~kW}\right)$; when managing power under the umbrella entity, no consumer exceeds the 
contracted power $\left(c_{1}^{u}=80 \mathrm{~kW}\right)$. A similar situation happens for $t=7$ and $t=8$. However, for $t=5$, the maximum demanded power of consumer three (60) cannot be flattened to $c_{2}^{u}$, although it is somehow diminished. The difference between $m_{2}^{u}-c_{2}^{u}(105-100)$ is shorter than $m_{2, k}-c_{2, k}(60-50)$, and so, depending on the $\beta_{k}$ value, the umbrella entity would be penalised or not, affecting the payment of consumer two. In case of the two consumers have exceed their allowed peak power at the same time, no re-allocation would have been carried out.

Regarding the payment, and assuming a power price $\pi_{i, k}=1$ for all $i, k$, $\alpha_{i, k}=0.85$, and $\beta_{i, k}=1.05$, consumer 1 out of the umbrella entity would pay, according to Equation 3,

- Period 1: $51 €$, since $1.05 * 40=42.00<m_{i, k}=45$

- Period 2: $45 €$, since $0.85 * 50=42.50<m_{i, k}=45<1.05 * 50=52.50$

- Period 3: $42 €$, since $1.05 * 30=31.50<m_{i, k}=35$

equalling a total of $138.00 €$. On behalf of consumer two, the payment would be,

- Period 1: $40 €$, since $0.85 * 40=34.00<m_{i, k}=40<42.00$

- Period 2: $75 €$, since $52.50<m_{i, k}=60$

- Period 3: $42 €$, since $31.50<m_{i, k}=35$

equalling a total amount of $157.00 €$. The sum of costs incurred without the umbrella entity is then $295.00 €$. On the other hand, according to Equation 6 and assuming $\alpha_{i, k}=\alpha_{k}=0.85$ and $\beta_{i, k}=\beta_{k}=1.05$, the umbrella entity would pay the following amounts for each period:

- Period 1: $78 €$, since $0.85 * 80=68.00<m_{i, k}=78<1.05 * 80=84.00$

- Period 2: $105 €$, since $0.85 * 100=85.00<m_{i, k}=105<1.05 * 100=$ 105.00

- Period 3: $55 €$, since $0,85 * 60=51.00<m_{i, k}=55<1.05 * 60=63.00$

Totalling the amount of $238.00 €$. The benefits are then considerable. The issue is how these benefits are shared among all the members of the coalition. 


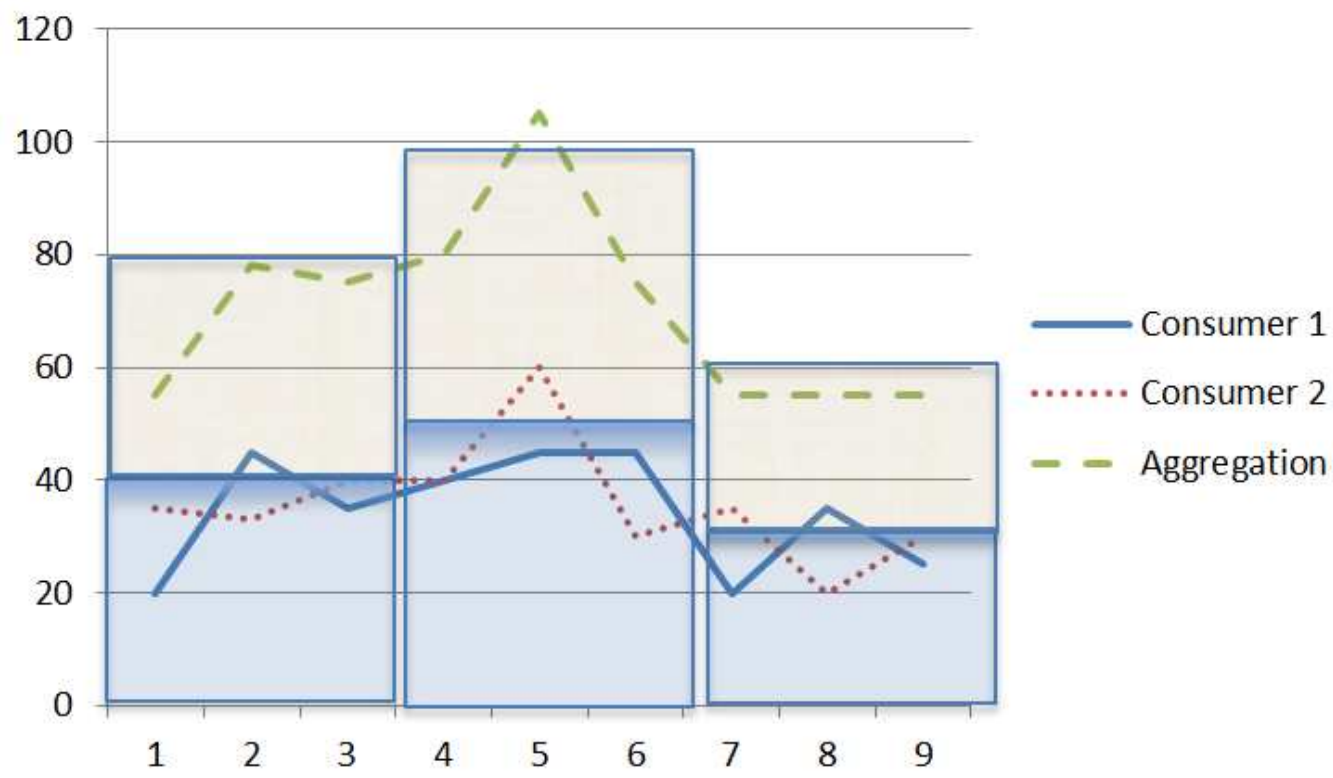

Figure 3: Example of energy consumption aggregation.

Current law forbids the resale of energy or power, thus the re-allocation of power needs to be done without an exchange of money. This fact limits the global saving that the community could achieve, i.e. a customer in the situation $\alpha_{i, k} c_{i, k} \leq p_{i, k, t}<\beta_{i, k} c_{i, k}$ could receive power from another one where $p_{i, k, t}>\beta_{i, k} c_{i, k}$ in exchange of a payment. The second customer will be penalised for its high power demand and thus it will be predisposed to pay, to the first consumer, to avoid these extra charges. However, due to law issues, we cannot follow this unconstrained coalition approach, and we constraint our solution to avoid money exchange.

Our proposal assumes agents collaboration, so that in some occasions one agent would receive power, while in other will give, with a common goal to reduce all of their costs. Each agent follows Equation 3 to compute their payment. The umbrella entity reallocates power among agents, so that the maximum demanded power by each agent $m_{i, k}$, for all $k$, is diminished due to the power exchange, so at the end, all agents have their costs reduced. Therefore, we guarantee that any consumer will pay less than running alone. Furthermore, the amount of money the umbrella entity will have to pay will be equal or lower than the sum of payments each customer would have to pay if they were running alone. 


\subsection{PRA basics}

PRA analyses the demanded power in repetitive slot series of the time window $W$, and seeks the power profiles that minimise power costs guaranteeing that no consumer will pay more than without PRA. The ideal situation happens when all consumers' demand is exactly $m_{i, k}=\alpha_{i, k} c_{i, k}$ according to the power costs expressed by Equation 2. If they demand a lower amount, they will pay the same; if they demand more, they will be penalised with extra charges.

Given the profiles of a set of customers, $p_{i, k, t} \forall i, k, t$, and their contracted power $c_{i, k}, \forall i, k$, PRA computes the new power profiles to minimise their maximum demanded power (Equation 3), meaning, they are all below $\alpha_{i, k} c_{i, k}$. To this end, power is shifted from one customer to another in order to keep each customer below the contracted power.

However, sometimes it is impossible to keep all demanded power at each slot $t$ below $\alpha_{i, k} c_{i, k}$. In such a situation, when at some slot $t$ a particular consumer has its maximum demanded power $m_{i, k}>\alpha_{i, k} c_{i, k}$ and it is impossible to reduce it, the consumer will have to pay, at least, for this $m_{i, k}$ along all the period $k$ of the time window $W$. That means that the consumer is only consuming the power it demands in a single slot, while in the remaining time window it is paying for power that is not used. To avoid such a situation, PRA increases the capacity of the consumer to receive power from others without increasing the amount of money it will pay.

To model this customer capacity we define the target power of each consumer $i$ for each period $k, \tau_{i, k}$, which is initialised to $\alpha_{i, k} c_{i, k}$. When at some point a particular consumer has its maximum demanded power $m_{i, k}>\tau_{i, k}$ and it is impossible to reduce it, PRA sets the target power to this new value $\tau_{i, k}^{\prime}=m_{i, k}$. The consumer will have to pay, at least, for this $m_{i, k}$ but it will have more capacity to accept demanded power from others.

Summarising, PRA is an iterative process, in which power reallocation and target power setting is repetitively applied until target powers become steady (see Figure 4). In our experiments a mean of 4.15 times with a standard deviation of 7.47 were sufficient in order to find the optimal reallocation.

On the other hand, re-allocation of power can be conducted according to different strategies, responding to a strategic decision of the trading company. In this work we propose a proportional strategy and two approaches based on priorities (received frequency and received amount strategies). In the 


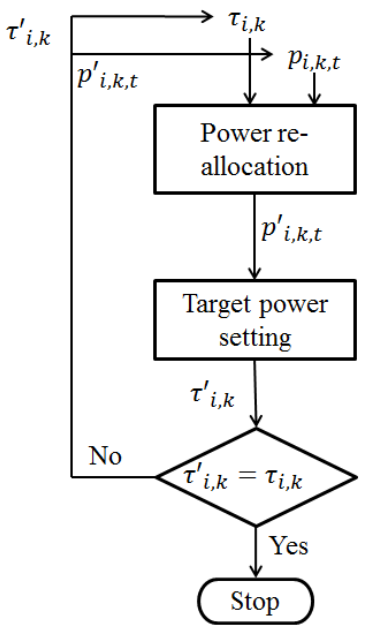

Figure 4: PRA iteration process

remainder of this section, we detail the steps of the algorithm, including the strategies.

\subsection{Power re-allocation step}

PRA tries to re-allocate power so that all consumers reach their target power. In doing so it determines that there are two kinds of roles among consumers:

- Receiver: if $m_{i, k}<\tau_{i, k}$. They receive power from other consumers.

- Giver: if $m_{i, k}>\tau_{i, k}$. They need to give power to other customers; otherwise they will pay extra charges due to extra demand.

First of all, it is important to know whether the addition of all the contracted powers is enough to keep every customer below its target power, in a given time slot $k$, considering the sum of all the power demanded by them. In doing this, we assume that the length of the slots reported by all the customers is the same. Note, however, that considering different time slots is an easy extension of our method by recursively applying the method with another scale. Then, we define:

Accumulated Power Rights: $A P R_{k, t}$ is the addition of all the contracted 
powers,

$$
A P R_{k, t}=\sum_{i=1}^{N_{c}} \tau_{i, k}
$$

Note that $A P R_{k, t}$ is calculated according to $\tau_{i, k}$ since it tells us the maximum power that a customer can demand without increasing the amount of money it has to pay.

Accumulated Demanded Power: $A D P_{k, t}$ is the addition of all the power demanded by consumers,

$$
A D P_{k, t}=\sum_{i=1}^{N_{c}} p_{i, k, t}
$$

Power Sharing $P S_{k, t}$ as the capacity of all customers to receive power from others without increasing their individual cost,

$$
P S_{k, t}=\sum_{i=1}^{N_{c}} \max \left(\tau_{i, k}-p_{i, k, t}, 0\right)
$$

If there is enough $A P R_{k, t}$, that is, $A D P_{k, t} \leq A P R_{k, t}$, then, there would be some power sharing to negotiate. The $P S_{k, t}$ is distributed by all the receivers according to some strategy.

Otherwise, if there is not enough $A P R_{k, t}$, that is, $A D P_{k, t}>A P R_{k, t}$ there would be some givers that cannot fulfil their target power. In spite of this, there could be givers and receivers, the latter offering power sharing (if $P S_{k, t}>0$ ), although not enough to cover all the demand. In this case, the givers can be classified into two categories:

- Non penalised customers: those who keep their demanded power between the target power and $\beta_{i, k} c_{i, k}$ (over-power demand).

- Penalised customers: those who exceed $\beta_{i, k} c_{i, k}$.

PRA first tries to reduce the demanded power of the penalised customers to lower them to either reach a value under $\beta_{i, k}$ otherwise the $P S_{k, t}$ is saturated (reaches 0$)$. After attending penalised receivers, if there is still power sharing available $\left(P S_{k, t}>0\right)$, then all the givers are treated according to the 


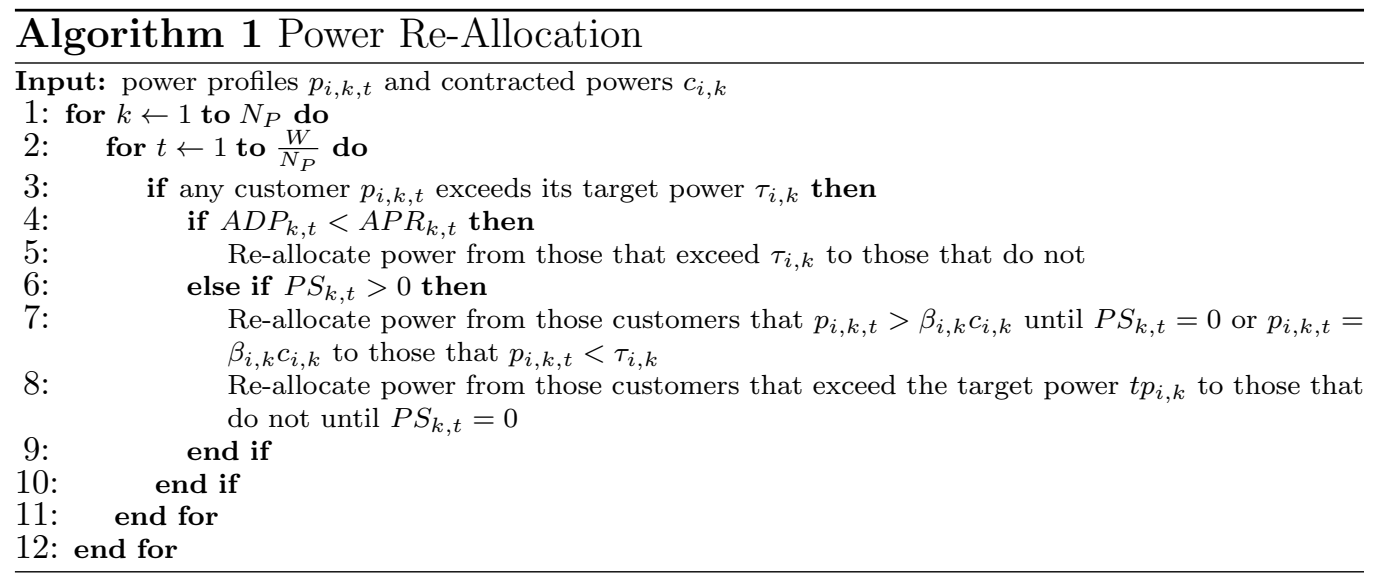

re-allocation strategy to lower their demanded power as much as possible. The power re-allocation algorithm is summarised at Algorithm 1.

If we consider that two profiles are complementary if when one exceeds its contracted power, the other is below $\alpha_{i, k} c_{i, k}$. Then we can say that the more complementary the demand profiles of the consumers are, the greater savings that PRA could achieve. Some examples of complementary profiles could be residential buildings with commercial or office buildings. However, even when demand profiles are very similar, PRA can achieve important benefits because it is scarcely possible that $m_{i, k}$ of each customer along the time window (i.e. a month) will correspond to the same time $t$. Besides, this method is not constrained to a maximum number of consumers, however, the addition of non-complementary consumers to a given coalition using PRA may reduce the individual savings but never the absolute global savings. Furthermore, this methodology is applicable to any kind of consumer which have a electricity billing depending on its maximum demanded power by using maximeters. Nevertheless, such kind of electricity bills is usually reserved to consumers with a contracted power greater than $15 \mathrm{~kW}$ (when maximeters are mandatory) while domestic contracted power is usually smaller than $5 \mathrm{~kW}$. Therefore, this excludes, for the time being, multi-family buildings with no centralised consumption accounting. However, the generalisation of smart meters, capable to measure the maximum demanded power, may change this fact. 


\subsection{Target power setting step}

Target power $\tau_{i, k}$ is initially set to $\alpha_{i, k} c_{i, k}$, and it is adjusted iteration after iteration. That is, after a PRA iteration, it calculates the new maximum demanded power $m_{i, k}^{\prime}$ for each consumer. If PRA does not achieve the ideal situation, in which all customers $m_{i, k}^{\prime} \leq \tau_{i, k}$, those which $m_{i, k}^{\prime}>\tau_{i, k}$ will be charged for the $m_{i, k}^{\prime}$ for all of period $k$. Therefore, $\tau_{i, k}$ is shifted closer to the new maximum, in order to increase the power sharing for the whole community. Notice that this does not increase $m_{i, k}$ and thus neither the payment.

In order to modify $\tau_{i, k}$, we need to take into account that some other customers could be in a similar situation. If they are requiring power in the same $t$, we can think of modifying $\tau_{i, k}$ according to the new $m_{i, k}^{\prime}$ value; but if their needs correspond to different $t$, a small increase in several customers could be enough to cover most of their needs. Therefore, we adjust $\tau_{i, k}$ according to the second maximum as follows:

$$
\tau_{i, k}^{n}=\max \left(\alpha_{i, k} c_{i, k}, \max _{\forall t \mid p_{i, k, t}<\max _{t}\left(p_{i, k, t}\right)}\left(p_{i, k, t}\right)\right)
$$

Observe, that the first iteration is equivalent to $\tau_{i, k}^{1}=\max \left(\alpha_{i, k} c_{i, k}, 0\right)$.

Going back to the example of Figure 3 and Subsection 4.1, the payment corresponding to each consumer using PRA would be:

- Consumer 1: $112.5 €$

- Period 1: $40 €$, since its final power profile at $k=3$ would be $p_{1,1, t}=[20,40,36]$ with $0.85 * 40=34<m_{1,1}=40<1.05 * 40=$ 42 .

- Period 2: 45€, since there is no power re-allocation because both peak power are at the same time and both exceed $0.85 * 50=42.5$.

- Period 3: $27.5 €$, since its final power profile at $k=3$ would be $p_{1,3, t}=[27.5,27.5,27.5]$ with $0.85 * 30=25.5<m_{1,3}=27.5<$ $1.05 * 30=31.5$.

- Consumer 2: $141.5 €$

- Period 1: 39€, since its final power profile at $k=3$ would be $p_{2,1, t}=[35,39,39]$ with $0.85 * 40=34<m_{2,1}=39<1.05 * 39=$ 42 . 
- Period 2: $75 €$, since there is no power re-allocation because both peak power are at the same time and both exceed $0.85 * 50=42.5$.

- Period 3: 27.5€, since its final power profile at $k=3$ would be $p_{2,3, t}=[27.5,27.5,27.5]$ with $0.85 * 30=25.5<m_{2,3}=27.5<$ $1.05 * 30=31.5$.

It is worth to point out that power re-allocation at periods 1 and 3 is possible thanks to re-set the target power of each consumer. Furthermore, the

total amount to pay by the two consumers rises to $254 €$ which is more than the $238 €$ of the umbrella entity. This fact is caused because no consumer has to pay more than running alone and so we limit power re-allocation. Then, in some situations there would be a surplus of money between the amount paid by the consumers respect the money paid by the umbrella entity which will have an extra income.

\subsection{Re-allocation strategies}

The distribution of power among givers and receivers can be performed following different strategies. The simple one is a proportional strategy; other strategies could take advantage of the exchange history. Along the PRA application, customers change their role from givers to receivers and vice versa according to their profiles and the APR. Therefore, it is possible to keep the history of each customer, and register how many times customers have received power from others. Fair strategies can use a priority model to prioritise customers that have often received power over other customers when they require to give power.

\subsubsection{Proportional re-allocation}

The power each receiver gets is proportional to the difference between its target power and its maximum demanded power. For example, consider that we have three customers with the same target power of $50 \mathrm{~kW}$ and their demanded powers at time $t$ are $41 \mathrm{~kW}, 44 \mathrm{~kW}$ and $60 \mathrm{~kW}$. So, the third customer gives $6 \mathrm{~kW}$ to the first customer (and then, its demanded power would be $47 \mathrm{~kW}$ ) and $4 \mathrm{~kW}$ to the second customer (and then, its demanded power would be $48 \mathrm{~kW}$ ).

Consider a second example in which there is not enough power sharing, where the three customers have the same target power equal to $50 \mathrm{~kW}$ and the same $\beta_{i, k} c_{i, k}=60$. Their respective demanded powers at time $t$ are $40 \mathrm{~kW}$, $55 \mathrm{~kW}$ and $65 \mathrm{~kW}$. The latter customer is greater than $\beta_{i, k} c_{i, k}$ and so it would 
be penalised. Thus, PRA re-allocates the power of this customer to the receiver converting their consumptions to $60 \mathrm{~kW}$ and $45 \mathrm{~kW}$ respectively. Since, there is still power sharing available it re-allocates again the power of all customers exceeding their target power to receivers, until $P S_{i, k}$ is saturated. Then the three corresponding demanded powers would be $50 \mathrm{~kW}$ (receiver), $53.33 \mathrm{~kW}$ and $56.67 \mathrm{~kW}$. Givers give an amount of power proportional to the difference between their demanded power and their target power.

\subsubsection{Received Frequency Priority (RFP)}

Along the PRA application, customers change their role from givers to receivers and vice versa, according to their profiles and the APR. Therefore, it is possible to keep the history of each customer, and register how many times customers have received power from others. This strategy prioritises customers that have often received power over other customers, when they are required to give power.

Priority is defined in $[0,1]$, where 0 represents the lowest priority and 1 the greatest.

Moreover, since power has different prices in different slots, it could be convenient to distinguish a priority per customer and period, priority $_{i, k}$. Given the time window $W$ in which PRA is applied, there are up to $W / N_{p}$ times that the same slot has been considered. On the other hand, we can define $x_{i, k}$ as the number of times it has received power from others in slot $k$. Then, the priority of each customer is calculated according to Equation (11).

$$
\text { priority }_{i, k}=\frac{x_{i, k}}{W / N_{P}}
$$

\subsubsection{Received Amount Priority (RAP)}

The aim of this method is to focus on the amount of energy received in the past, instead of the frequency. To this end, we need to be aware of the maximum capacity that any customer can receive, i.e, $\alpha_{i, j} c_{i}$.

We define $z_{i, k, t}$ as the amount of power that customer $i$ has received from others, at instance $t$ in period $k$. Consistently, priorities are calculated according to Equation (12).

$$
\text { priority }_{i, k}=\frac{\sum_{t=1}^{W / N_{P}} z_{i, k, t} /\left(\alpha_{i, k} c_{i}\right)}{W / N_{P}}
$$


Priorities can also be set according to customer types or according to other strategies that can be studied in further work.

\section{Experimentation}

In this section we first explain the experimental setup over which we conducted the experimentation. Then we study and analyse the performance of the methodology and reallocation strategies presented in this paper.

\subsection{Experimental set up}

The data on which we conducted our experiment is based on the real electric energy consumptions of eight business buildings (office and teaching buildings of the University of Girona) $\left(N_{c}=8\right)$. We analysed the data in order to estimate the probability distributions of the power consumptions in a day (differentiating between workdays and vacations/weekend) ${ }^{2}$. From that distribution, we get the $p_{i, k, t}$ of each consumer. Figure 5 shows the box plot of the demanded power of each customer on workdays. We set $N_{p}=3$ because this is the current number of periods of the Spanish model.

We set the contracted power of each customer $\left(c_{i, k}\right)$ to the optimal one, which can be obtained since we know all the historical information of the simulated companies. That is, for each period $k$, it is possible to know what is the maximum demanded power of a consumer, and then, assign that as the contracted power, meaning than the derived cost will be the best according to Equation 3. Of course, this is the optimal power regarding the consumers work in isolation; we expect to improve this value when consumers work in coalition. Dealing with the optimal contracted power ensures that the results of the different methods are comparable. Regarding the $\alpha i, k$ and $\beta_{i, k}$, they have been set to 0.85 and 1.05 correspondingly.

Regarding power prices, we considered three different periods for all customers and the same prices for each customer: $3.31 € / \mathrm{kW}$ for $k=1,1.98$ $€ / \mathrm{kW}$ for $k=2$ and $1.32 € / \mathrm{kW}$ for $k=3$. These prices have been taken from current companies bills.

In order to analyse PRA and the reallocations strategies, we conducted experimentation over two different scenarios:

\footnotetext{
${ }^{2}$ Probability density functions available at http://eia.udg.es/ ${ }^{\sim}$ ftorrent/powerProfiles.pdf
} 
- Scenario 1: Analysis and comparison of costs regarding the benefits of using PRA. To this end, three configurations are considered,

- no-PRA: the consumers manage power by themselves

- Aggregation: an unconstrained umbrella entity which pays for all of the consumers, and proportionally shares the power.

- PRA: an umbrella entity with PRA using the proportional strategy

Simulations are run for one month of the electric consumption of each customer. Our hypothesis is that either the use of PRA or the aggregation will convey a reduction of the cost compared with no-PRA. Moreover, with PRA, consumers are guaranteed that will never pay more than alone.

- Scenario 2: in this second scenario we study the implications of using different reallocation strategies. We conducted experimentation for one simulated year because one month is a too short period of time to achieve significant differences concerning the amount of power or how many times customers receive power from others. In addition to the RAP, the RFP and the proportional priority strategy we defined a random priority strategy. We expect that RAP and RFP will behave better than the random and proportional strategy.

\subsection{Results and Discussion}

Results are analysed on the basis of the following measures:

- Global costs $(€)$ : the sum of the power costs of all customers according to Equation (2),

$$
\sum_{i, k} \operatorname{cost}\left(m_{i, k}\right)
$$

- Customer costs $(€)$ : the sum of the power costs for a customer $i$ inside a time window,

$$
\sum_{k} \operatorname{cost}\left(m_{i, k}\right)
$$



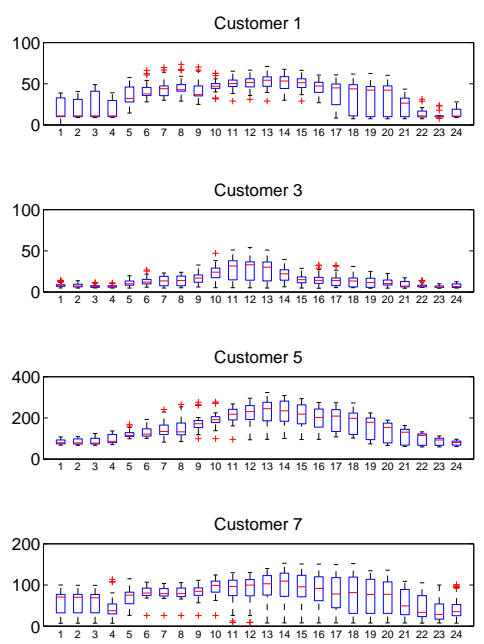
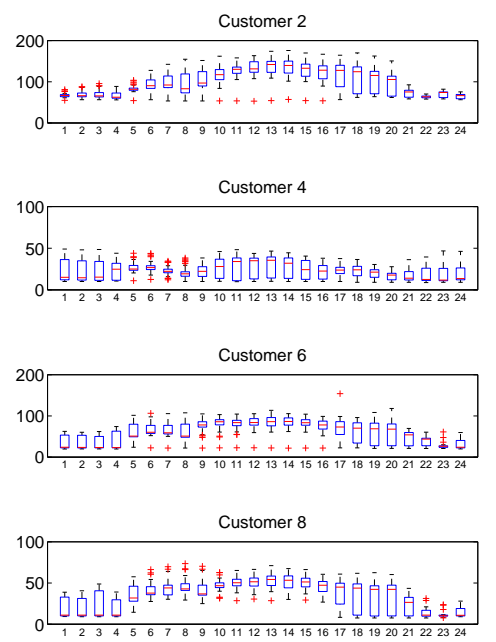

Figure 5: Box plots of the demanded power of each customer along workdays.

- Final power profile $(\mathrm{kW})$ : the power required by a particular customer $i$ as a result of the method (final $p_{i}$ )

- Gini coefficient: to evaluate the fairness of each strategy, we used the Gini coefficient [4] taking as wealth the savings of each customer, and as size of the population the mean power that each customer receives from others. Thus we calculate the Gini coefficient, weighting the saving of each customer by the power it has received from others. This means that the index is a measure of fairness regarding the benefits of each customer with to respect how useful it has been to the other customers.

Results are provided in average after 100 repetitions of the simulations.

Scenario 1: Benefits of using PRA. As a first example, Figure 6 shows the target power of four customers $\left(\tau_{i, k}\right)$ and the final power profile of each one along a day when using PRA and when not ${ }^{3}$. Figure 6 shows how demanded power for those customers that exceed their contracted power, is re-allocated

\footnotetext{
${ }^{3}$ At $t=20$ the other customers give or receive small amounts of power and we do not show them.
} 

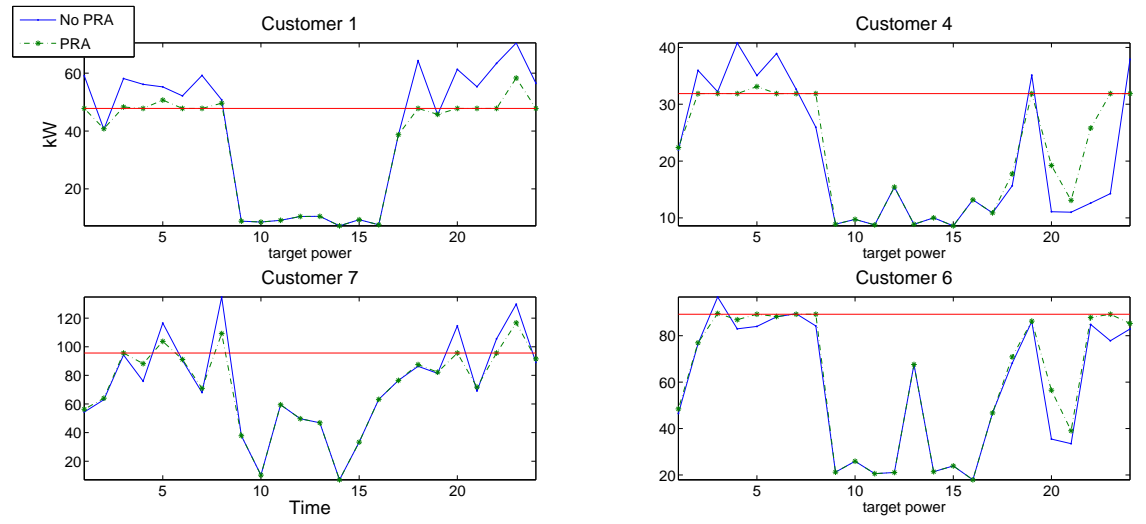

Figure 6: Power profiles along a day of four customers using PRA and without PRA compared with the target power of each one (flat line)

to other customers. For example, at $t=20$ customers one and seven demand a power of $61 \mathrm{~kW}$ and $113 \mathrm{~kW}$. If no re-allocation is performed, demanded power will exceed contracted power (and target power); but when using PRA, they can reduce demanded power to $47 \mathrm{~kW}$ and $96 \mathrm{~kW}$ respectively, which keeps them below their power target. On the other hand, customers four and six have a demanded power of $11 \mathrm{~kW}$ and $35 \mathrm{~kW}$ respectively, but after re-allocation their demanded power increases to $19 \mathrm{~kW}$ and $57 \mathrm{~kW}$. Therefore, PRA follows a peak shaving and valley filling strategy for the power required by each customer but it does not change the overall demand.

Note that customers that receive power from others never surpass their target power and therefore they do not increase their own power costs.

Figure 7 shows the global costs of eight customers with their optimal contracted power $(\mathrm{x}=1.0)$, and other costs resulting from adjusting the contracted power upwards (1.05*optimal power, $1.10 *$ optimal power, ...) and downwards $(0.95 *$ optimal power, ...). Figure 7 shows that in general, using PRA or the aggregation configuration achieves a great reduction in the cost of the whole demand, with respect to the case where each customer has its own contracted power and there is not a re-allocation of the demanded power (non-PRA). Comparing PRA with the aggregation configuration, we see that as expected, the aggregation obtains the minimum possible cost for each possible contracted power. However, when customers have their optimal contracted power for their demand, the difference between PRA and the aggregation is negligible $\left(5.1 \cdot 10^{3}\right.$ and $5.05 \cdot 10^{3}$ correspondingly). That point 
means that PRA is able to achieve the minimal possible cost when customers have their optimal contracted power. In other situations, it is important to note that the aggregation does not guarantee that the consumer will pay less than in an individual way, whereas PRA does.

Focusing on each customer, Figure 8 shows the cost each customer would pay for each method (PRA, no-PRA and aggregation). We see that PRA guarantees that any customer will not increase its cost while aggregating the demands does not guarantee it. For example, customers five and seven achieve their lowest cost when PRA is used. The cost reduction for each customer goes from $14.63 \%$ (customer five) to $24.29 \%$ (customer eight). Thus each customer achieves an important reduction of the cost.

It is worth pointing out that PRA has been tested with eight different customers that share a similar power profile (the highest peaks are approximately at the same time each day, and they have vacations, valleys, at the same time). However PRA is more effective if profiles are complementary (peaks of one customer correspond to a valley of another). Even in such conditions the cost of reduction is very important, around $20 \%$ in average. Thus the savings achieved by PRA may be an incentive for industrial parks or other communities of customers to tend to complementary profiles.

Results in Figure 7 also show that PRA becomes useless when the contracted power of each customer is sufficiently high (worse case for $\mathrm{x}=1.50$ of the optimal contracted power) and no re-allocation is needed. It also shows a trend indicating that for very low contracted powers (worse case for $\mathrm{x}=0.50$ of the optimal contracted power) the cost increases and tends to be equal to the cost with No-PRA. This is because most of the time the customers' demands exceed their contracted power, and power re-allocation is not possible.

The main benefit of PRA comes from the fact that it returns information regarding the possibility of reducing contracted power. From Figure 7 we see that the cost curve reaches the minimum when using PRA at $\mathrm{x}=0.80$, meaning, that all customers could propose a new contract power for that value to the company. Comparing the minimum No-PRA cost with the minimum PRA cost, we see that there is a $20 \%$ reduction. In the real case under study, this means a saving of around $1300 €$ per month for the overall cost. The investment and costs required for our methodology is the implantation of smart meters (300€ per unit) to all consumers (which are currently being implanted in many countries) and additional one for the umbrella entity to be able to measure the aggregated consumption. The cost of managing the umbrella entity we think is negligible if it is managed by an electricity trade 


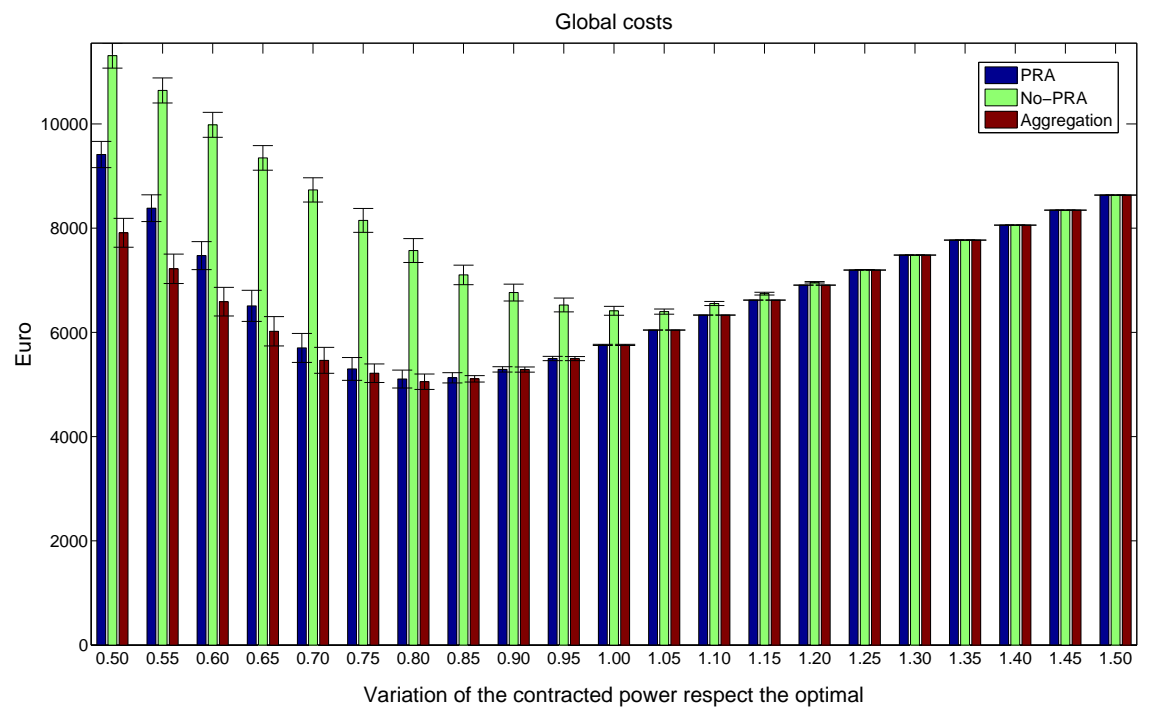

Figure 7: Average and standard deviation of the sum of power costs of all customers modifying all $c_{i, k}$ proportionally respect the contracted power, $c_{i, k}^{o}$, that minimizes PRA power costs.

company. Given these costs and these experimental results we can say that the benefits of using PRA widely surpass its costs.

On the other hand, the reduction of the contracted power not only benefits consumers, but also electricity companies. When a consumer has a particular contracted power, the electricity distribution company has the duty to satisfy a power demand of this value at any time. Thus, an increase of the contracted power by the consumers (or an increase of the number of consumers) conveys an adjustment of the grid, even if this grid is underutilised most of the time. In this sense, a reduction of the contracted power by the consumers, without reducing the demand, increases the utilisation of the grid, benefiting electricity companies because they can make the most of their infrastructure.

Scenario 2: Priority trade-off. Experimentation with Scenario 2 showed us that global costs do not depend on the priority strategy used. This was an expected result because the priority strategies are only used to decide the amount of energy each customer can give when it is impossible to keep all of them below their target power. Thus, we think it is important to have a strategy that guarantees some fairness, not in terms of equity, but in terms of benefiting those customers that are active receivers. 


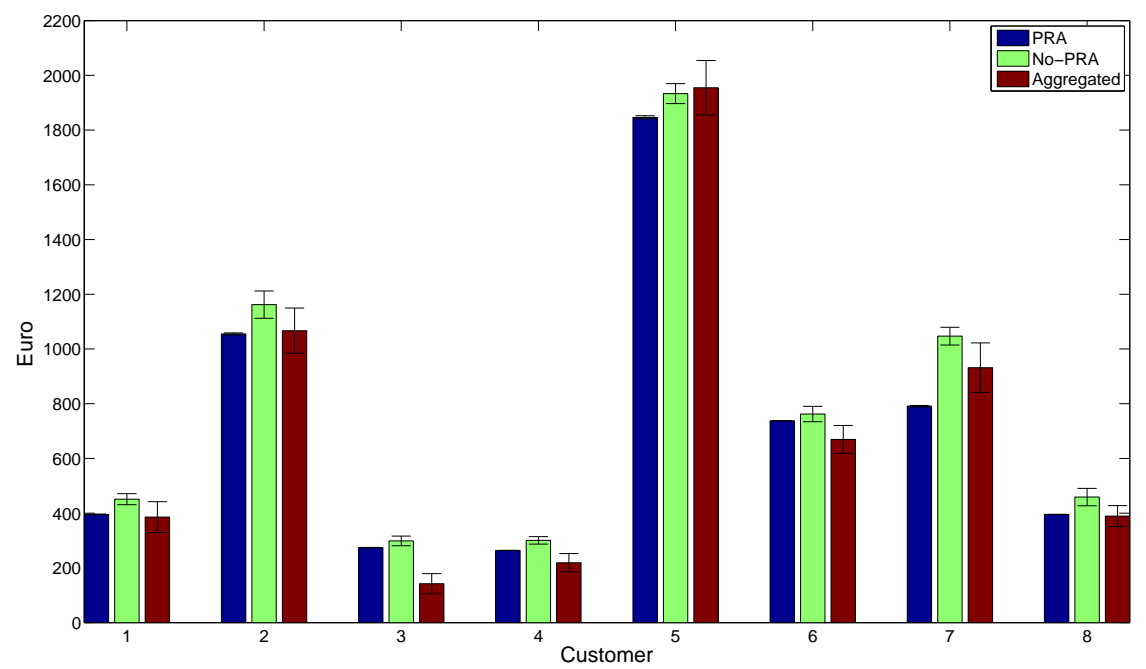

Figure 8: Power cost of each customer.

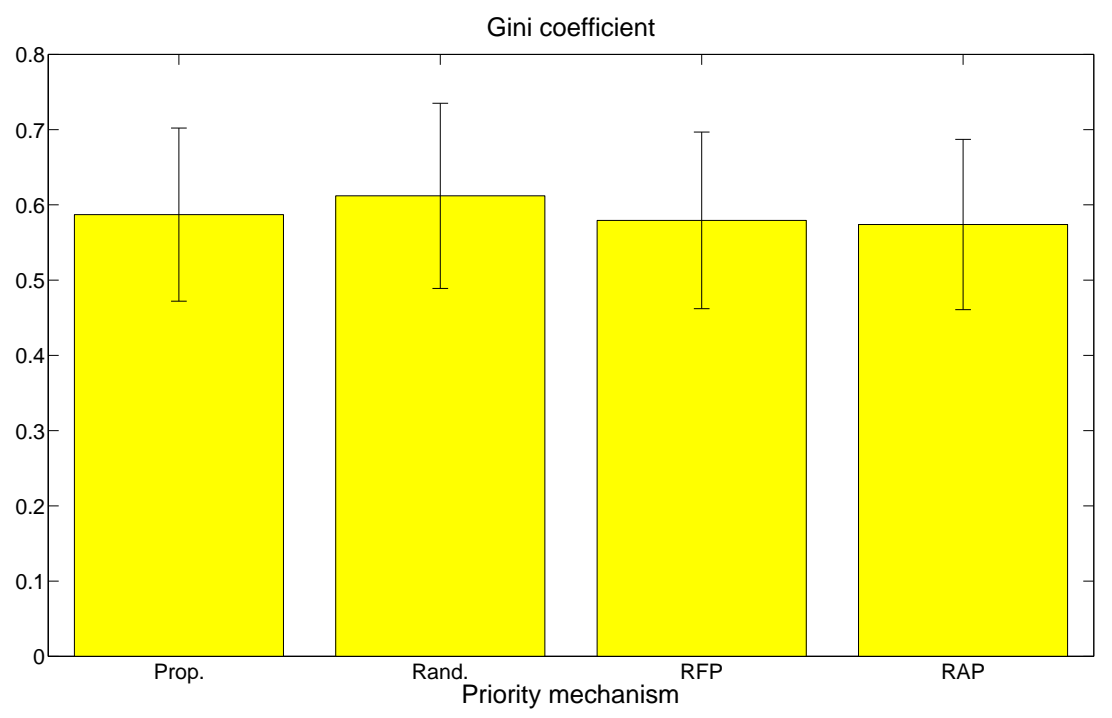

Figure 9: Gini coefficient achieved by each priority strategy. On the left the wealth was set as the savings achieved by each consumer and the population size was set as the amount of received power. On the right the wealth was set as the savings and the population size was set as the number of times each customer received power. 
Figure 9 shows the Gini coefficient (the mean and the standard deviation) achieved by each priority strategy. It shows that the use of RFP or RAP reduces the Gini coefficient. Since we are calculating the Gini index, taking as the wealth the relative savings of each consumer, and the size of each population as the amount of received power by each customer, the index indicates the fairness of each strategy. According to this index a fair strategy is the one that provides, each consumer with a relative saving proportional to the amount of received power. In this way, Figure 9 shows that RFP and RAP strategies are fairer than the others. Particularly, RAP is the fairest strategy.

We have also computed paired-response (with a significance level of 5\%) tests of the Gini indices with 100 repetitions of the experiment and these tests show that the RAP strategy does achieve the lower Gini index, followed by RFP. Pair-response tests also conclude that using random priorities performs worse than using the proportional method.

\section{Conclusions and future work}

Electricity companies invoice their customers with their maximum power demand peak and, depending on whether such a peak exceeds their contracted power, penalising their consumers with extra charges. This paper presents a method for re-allocating the power demand by a coalition of consumers, in order to avoid extra charges due to the demanded power as well as the contracted power. In doing so, we avoid an exchange of money between the consumers and guarantee that no consumer will have a greater cost due to such power re-allocation. This methodology needs a (virtual) umbrella entity that groups consumers and carries out power re-allocation.

The paper presents some priority strategies in order to benefit those consumers that accept receiving demanded power from others. In this way, we propose a method that uses solidarity among consumers to reduce power costs but includes strategies of fairness that guarantee that no consumer will be charged for an excess of demand of another consumer, and that those that accept more power from others will be treated in a preferential way.

Finally we analysed the performance of the methodology presented in the paper using the real-based simulated consumptions of eight buildings. The results showed that re-allocation reduces power costs by about 20\% (surpassing the costs of carrying it out) and allows a reduction of the contracted power by the consumers without increasing penalties. This latter result is 
also positive for electricity companies because it would help to increase the utilisation index of the grid, because consumers would be able to reduce their contracted power without increasing penalties for exceeding it, and thus, the power grid will be able to accept more consumers (in an increasing electricity demand scenario) without extra investments. Then, a generalised reduction of the contracted powers, besides increasing the network usage, may involve a reduction of active and installed generators, reducing human pressure to Earth resources.

It is important to point out that the more different the demand profiles of the consumers are, the greater savings that PRA will achieve. However, even when demand profiles are very similar, PRA can achieve important benefits because it is scarcely possible that maximum electricity demand of each customer along the time window (i.e. a month) corresponds to the same time. Nevertheless, a future research should be guided towards quantifying how many buildings or industries of each kind of power profile would be the optimum, as well as, mapping power profiles and the power grid using geographic information systems to work out the areas with a greatest potential benefit for using power re-allocation. Furthermore, this information could be used for local energy and land use planning in order to flatten aggregated power profiles by placing complementary consumers close to each other and hanging from the same line of the power grid.

Regarding the improvement of the methodology, there are interesting lines

of research as the study of other priority strategies or the implications of the cold start and the arrival of new customers.

\section{Acknowledgements}

This research project has been partially funded through BR-UdG Scholarship of the University of Girona granted to Ferran Torrent-Fontbona. Work developed with the support of the research group SITES awarded with distinction by the Generalitat de Catalunya (SGR 2014-2016) and the MESC project funded by the Spanish MINECO (Ref. DPI2013-47450-C2-1-R).

\section{References}

[1] Bakari, K. E., \& Kling, W. L. (2010). Virtual power plants: An answer to increasing distributed generation. In 2010 IEEE PES Innovative Smart Grid Technologies Conference Europe (ISGT Europe) (pp. 1-6). 
[2] Brounen, D., Kok, N., \& Quigley, J. M. (2013). Energy literacy, awareness, and conservation behavior of residential households. Energy Economics, 38, 42-50.

[3] Faria, P., Vale, Z., Soares, J., \& Ferreira, J. (2011). Demand response management in power systems using a particle swarm optimization approach. IEEE Intelligent Systems, 28, 43-51.

[4] Gastwirth, J. (1972). The estimation of the Lorenz curve and Gini index. The Review of Economics and Statistics, 54, 306-316.

[5] Gottwalt, S., Ketter, W., Block, C., Collins, J., \& Weinhardt, C. (2011). Demand side management - A simulation of household behavior under variable prices. Energy Policy, 39, 8163-8174.

[6] Jia, W., Kang, C., \& Chen, Q. (2012). Analysis on demand-side interactive response capability for power system dispatch in a smart grid framework. Electric Power Systems Research, 90, 11-17.

[7] Ketter, W., Collins, J., \& Reddy, P. (2013). Power TAC: A Competitive Economic Simulation of the Smart Grid. Energy Economics, 39, 262270.

[8] Kwag, H.-G., Kim, Y.-H., \& Kim, J.-O. (2012). Virtual peak plant concepts of Demand Response. In Systems and Informatics (ICSAI), 2012 International Conference on (pp. 614-618).

[9] Law, Y. W., Alpcan, T., Lee, V., Lo, A., Marusic, S., \& Palaniswami, M. (2012). Demand response architectures and load management algorithms for energy-efficient power grids: a survey. In Knowledge, Information and Creativity Support Systems (KICSS), 2012 Seventh International Conference on (pp. 134-141).

[10] Leng, M., Parlar, M., \& Zhang, D. (2014). Cooperative game analysis of retail space-exchange problems. European Journal of Operational Research, 232, 393-404.

[11] Meir, I.A. \& Pearlmutter, D. (2010). Building for climate change: planning and design considerations in time of climatic uncertainty. Corrosion Engineering Science and Technology, 45, 70-75. 
[12] Meir, I.A., Peeters, A., Pearlmutter, D., Halassa, S., Garb, Y.,\& Davis, J.M. (2012). Green building standards in MENA: An assessment of regional constraints, needs and trends. Journal Advances in Building Energy Research, 6, 173-211.

[13] Mohsenian-Rad, A.-H., Wong, V. W. S., Jatskevich, J., Schober, R., \& Leon-Garcia, A. (2010). Autonomous Demand-Side Management Based on Game-Theoretic Energy Consumption Scheduling for the Future Smart Grid. IEEE Transactions on Smart Grid, 1, 320-331.

[14] Pudjianto, D., Ramsay, C., \& Strbac, G. (2007). Virtual power plant and system integration of distributed energy resources. Renewable power generation, IET , 1, 10-16.

[15] Simonis, H., \& Hadzic, T. (2011). A Resource Cost Aware Cumulative. In J. Larrosa, \& B. O'Sullivan (Eds.), Recent Advances in Constraints ecent Advances in Constraints (pp. 76-89). Heidelberg: Springer Berlin. (Lecture no ed.).

[16] Torrent-Fontbona, F., \& López, B. (2013). Comparison of Workflow Scheduling Using Constraint Programming or Auctions Research Report 13-01-RR. Technical Report March Institute of Informatics and Applications, University of Girona Girona.

[17] Vinyals, M., Bistaffa, F., \& Rogers, A. (2012). Stable coalition formation among energy consumers in the smart grid. In Proceedings of the 3rd International Workshop on Agent Technologies for Energy Systems (ATES 2012) i. Valencia.

[18] Zhang, Z., Kusiak, A., \& Song, Z. (2013). Scheduling electric power production at a wind farm. European Journal of Operational Research, 224, 227-238. 